\title{
Bone scan use in the management of metastatic castration-resistant prostate cancer: Survey of practice patterns among Canadian radiation oncologists, medical oncologists, and urologists
}

\author{
Mahbuba Meem ${ }^{1}$; Katherine Zukotynski ${ }^{2 *}$; Srinivas Raman ${ }^{3,4}$; Urban Emmenegger ${ }^{*}$ \\ ${ }^{1}$ Faculty of Medicine, University of Toronto, Toronto, ON, Canada; ${ }^{2}$ Departments of Medicine and Radiology, \\ McMaster University, Hamilton, ON, Canada; ${ }^{3}$ Radiation Medicine Program, Princess Margaret Cancer Centre, \\ Toronto, ON, Canada; ${ }^{4}$ Department of Radiation Oncology, University of Toronto, Toronto, ON, Canada; \\ ${ }^{5}$ Sunnybrook Odette Cancer Centre and Research Institute, University of Toronto, Toronto, ON, Canada \\ *Equal contributors
}

Acknowledgements: The authors would like to thank all physicians and professional societies (GUMOC, GUROC, CUOG, CARO) who participated in the survey. The survey was made possible by financial support from the Genitourinary Cancer Site Group at Odette Cancer Centre, Sunnybrook Health Sciences Centre (Toronto, Canada). Furthermore, the research team of Dr. Emmenegger is supported by the Joseph and Silvana Melara Cancer Fund.

Cite as: Can Urol Assoc J 2020 June 5; Epub ahead of print. http://dx.doi.org/10.5489/cuaj.6320

Published online June 5, 2020

$* * *$

\section{Introduction}

The use of skeletal scintigraphy with technetium-99 methylene diphosphonate (hereafter referred to as a bone scan) for evaluating response to systemic treatment in men with metastatic castration-resistant prostate cancer (mCRPC) is an evolving paradigm in this era of advancing therapies and imaging techniques. Indeed, the interpretation of bone scans can be challenging, and there is a growing expectation that advanced imaging techniques such as prostate-specific membrane antigen positron emission tomography/computer tomography (PSMA PET/CT) may play a complementary role. ${ }^{1}$ The Prostate Cancer Working Group (PCWG) has outlined specific criteria to define disease progression with respect to bone scans performed as part of clinical trials. ${ }^{2}$ However, there is no high-level evidence for the scheduling and interpretation of bone scans during routine therapeutic interventions for mCRPC. Thus, patterns of bone scan use are variable and practice-dependent outside of clinical trials.

\section{Methods}

In this survey approved by the Research Ethics Board of Sunnybrook Health Sciences Centre (Toronto, Canada) we sought to understand practice patterns of bone scan use in the management of mCRPC among Canadian radiation oncologists, medical oncologists and urologists as well as their experience with new imaging techniques. A letter of invitation 
including a description of study objectives and an embedded web link to complete the survey was distributed through the internal emailing lists of the Genitourinary Medical Oncologists of Canada (GUMOC), Genitourinary Radiation Oncologists of Canada (GUROC), Canadian Urological Oncology Group (CUOG), and Canadian Association of Radiation Oncology (CARO). The survey was administered through SurveyMonkey ${ }^{\circledR}$ (Palo Alto, CA, USA) for anonymous submission. A gift certificate was offered to each respondent at the completion of the survey. The first set of invitations was sent on 27 March 2018 and the survey remained active for 7 months. Responses were analyzed using descriptive statistics in the form of frequencies and percentages. Responses to rank order questions were analyzed comparatively using stacked bar charts.

\section{Results}

We had a total of 91 participants in our survey consisting of $45.0 \%$ radiation oncologists (41/91), 37.4\% medical oncologists (34/91), and 17.6\% urologists (16/91). Most were from Ontario (53.3\%) and British Columbia (24.4\%), working in an academic setting (75.8\%), and treating either $10-25$ patients ( $40.4 \%$ of respondents), $25-50$ patients $(36.0 \%)$, or $>50$ mCRPC patients $(23.6 \%)$ in a given year.

While $94.3 \%$ of respondents indicated they would order a baseline bone scan prior to initiating a new line of systemic therapy, about half (51.7\%) replied they would forgo scheduling bone scans in asymptomatic men on treatment (Table 1). One in five indicated they would order bone scans in asymptomatic men if the PSA doubling time was alarming. The percentages of physicians who routinely schedule a bone scan every 3-4 months, 6 months or 12 months in men on therapy were $2.2 \%, 13.5 \%$ and $12.4 \%$, respectively, largely independent of the treatment used. Almost half of respondents (47.7\%) confirmed signs of progression on a bone scan with additional imaging, with one third (32.6\%) ordering a follow-up bone scan to exclude a potential flare phenomenon.

Symptoms (72.7\%) and rising PSA $(60.7 \%)$ were the two most frequently cited triggers for ordering a non-scheduled bone scan.

When asked to rank several measures of treatment response in order of clinical significance, symptomatic progression and skeletal related events were ranked most commonly in the top two 2 (Figure 1). Most respondents (80.4\%) ranked bone scan progression as less important (ie $3^{\text {rd }}$ to $5^{\text {th }}$ position for clinical significance).

To determine bone scan related progression, $81 \%$ of participants wrote they rely on the wording of the bone scan report, with $64 \%$ analyzing the bone scans themselves, $24 \%$ using PCWG3 criteria, and 9\% correlating bone scan findings with sites of symptomatic disease. Only $1 \%$ use the bone scan index. ${ }^{3}$

Routine use of advanced imaging such as PSMA, 18F-NaF and 18F-fluciclovine PET/CT was low at the time of the survey: $2.2 \%(2 / 89), 1.1 \%(1 / 89)$, and $0 \%(0 / 89)$ of participants, respectively.

\section{Discussion}

In men with mCRPC to bones accurate, easily accessible and validated biomarkers of 
response remain enigmatic, and clinical guidelines for assessing response to systemic therapy are relatively vague and heterogenous. ${ }^{4-7}$ Hence, it is perhaps not astonishing that our survey shows significant variability in how bone metastases are monitored and how progression is defined.

While bone scan reports often subjectively indicate if there is a change in the burden of disease, they may fail to precisely quantitate the disease burden, thus rendering the report valuable primarily for identifying "progression", "stable disease/no progression" and "response". To distinguish between flare and progression on treatment the PCWG3 has defined the latter as 'At least two new lesions on first post-treatment scan, with at least two additional lesions on the next scan $(2+2$ rule $)$ '. ${ }^{2}$

Currently, mCRPC progression is typically defined by clinical symptoms, PSA changes and imaging (both in bone and soft tissue), with high clinical significance attributed to symptoms ${ }^{5}$. Likewise, our respondents viewed progression on bone scans in asymptomatic patients as a less relevant indicator of progression when making treatment decisions. On the other hand, the analysis of two large phase III trials suggests that radiographic progressionfree survival in men with mCRPC (using PCWG3 criteria) is a robust surrogate for overall survival. ${ }^{8-9}$

Historically, the mainstay of treatment for men with mCRPC has been systemic therapy, and the question of progression and when to switch treatment has been a binary choice. Presumably, changes in PSA levels and symptomatic progression would suffice then. However, recent advances suggest that there might be an important opportunity to treat men with oligometastatic prostate cancer with metastasis directed therapy (MDT). ${ }^{10}$ This approach is supported by encouraging results from the SABR-COMET (all cancers) ${ }^{11}$, STOMP (castration-sensitive oligorecurrent prostate cancer) ${ }^{12}$ and ORIOLE (castration-sensitive oligometastatic prostate cancer) ${ }^{13}$ phase II clinical trials. Furthermore, there are several ongoing phase III clinical trials seeking to definitively demonstrate the benefit of MTD in prostate cancer, including two Canadian studies: PLATON/PR.20 [NCT03784755], and PCS IX [NCT02685397]. The results of these trials may further guide how closely we want to follow mCRPC to bone.

The results of our study should be interpreted in light of some limitations, including the relatively small sample size, ineffectiveness in capturing nuanced responses through close-ended questions, and underrepresentation of community practitioners treating mCRPC.

\section{Conclusions}

Consistent with the lack of consensus among clinical guidelines our findings provide evidence of marked variation in practice around scheduling bone scans for assessing treatment response and disease progression in men with mCRPC. Physicians rely predominantly on change in symptoms for therapeutic guidance. Encouraging results from recent trials treating oligometastatic disease with MDT and ongoing Canadian trials exploring the benefit of MDT in men with prostate cancer may result in a fundamental change in the treatment paradigm of mCRPC. Arguably, therapy of mCRPC may shift from a systemic approach to one where systemic agents and MDT are combined for improved patient 
survival. Hence, accurately identifying the burden of disease in men with mCRPC, even in asymptomatic patients, could emerge as a crucial step in management.

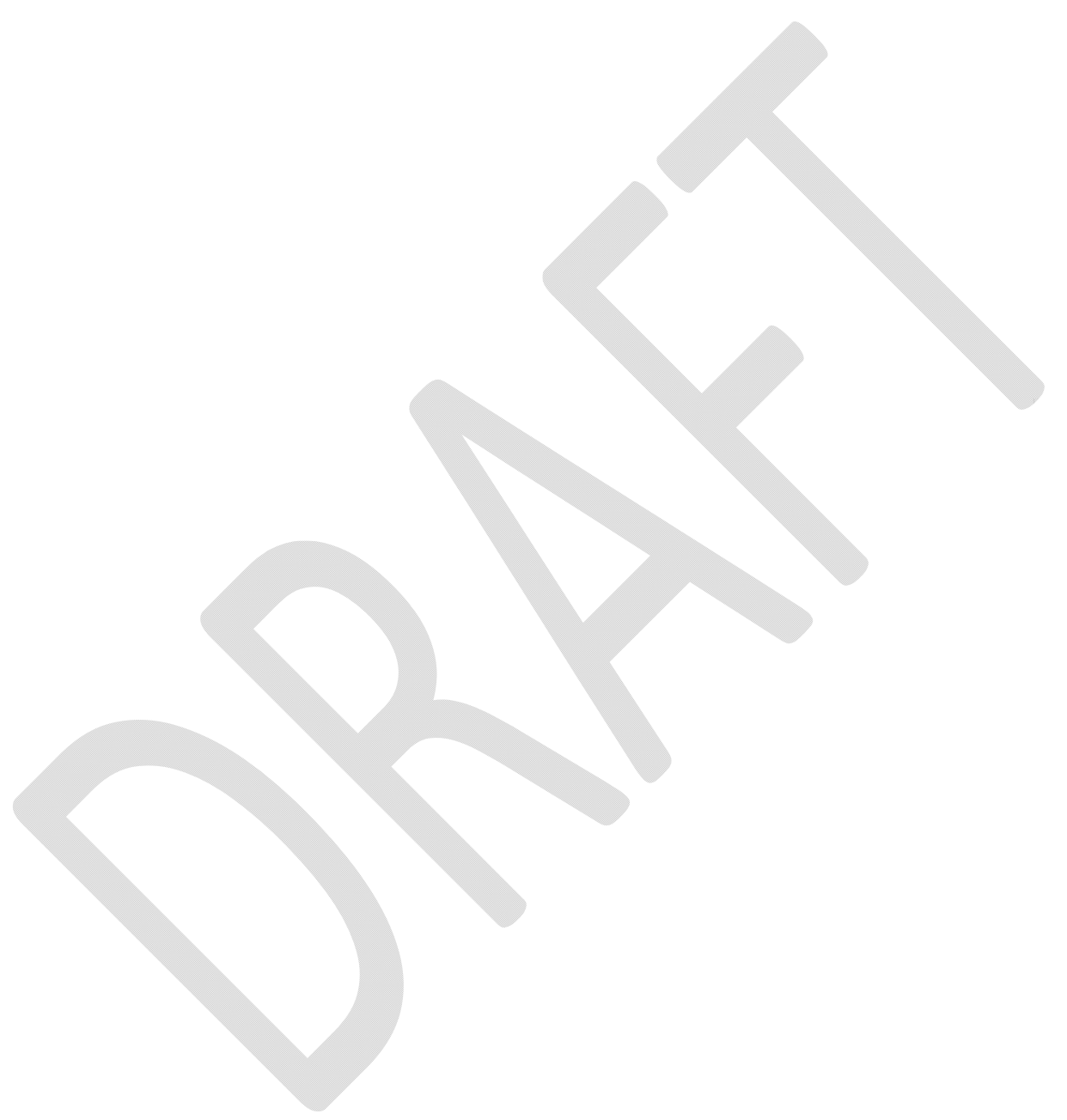




\section{References}

1. Armstrong A, Anand A, Edenbrandt L, A et al. Phase 3 assessment of the automated bone scan index as a prognostic imaging biomarker of overall survival in men with Metastatic Castration-Resistant Prostate Cancer. JAMA Oncol 2018; 4:944-951.

2. Scher H, Morris M, Stadler W, et al. Trial design and objectives for castrationresistant prostate cancer: Updated recommendations from the prostate cancer clinical trials working group 3. J Clin Oncol 2016; 34:1402-18.

3. Dennis E, Jia X, Mezheritskiy I, et al. Bone scan index: A quantitative treatment response biomarker for castration-resistant metastatic prostate cancer. J Clin Oncol 2012; 30:519-24.

4. Parker C, Gillessen S, Heidenreich A, et al. Cancer of the prostate: ESMO clinical practice guidelines for diagnosis, treatment and follow-up. Ann Oncol 2015;26: v6977.

5. Gillessen S, Attard G, Beer T, et al. Management of patients with advanced prostate cancer: The report of the advanced prostate cancer consensus conference APCCC 2017. Eur Urol 2018; 73:178-211.

6. Saad F, Aprikian A, Finelli A, et al. 2019 Canadian Urological Association (CUA)Canadian Uro Oncology Group (CUOG) guideline: Management of castrationresistant prostate cancer (CRPC). Can Urol Assoc J 2019; 13:307-314.

7. Cornford P, Bellmunt J, Bolla M, et al. EAU-ESTRO-SIOG guidelines on prostate cancer. Part II: treatment of relapsing, metastatic, and castration-resistant prostate cancer. Eur Urol 2017; 71:630-642.

8. Rathkopf D, Beer T, Loriot Y, et al. Radiographic progression-free survival as a clinically meaningful end point in metastatic castration-resistant prostate cancer: The PREVAIL Randomized Clinical Trial. JAMA Oncol 2018; 4:694-701.

9. Morris M, Molina A, Small E, et al. Radiographic progression-free survival as a response biomarker in metastatic castration-resistant prostate cancer: COU-AA-302 results. J Clin Oncol 2015; 3:1356-63.

10. Saluja R, Cheung P, Zukotynski K, et al. Disease volume and distribution as drivers of treatment decisions in metastatic prostate cancer: From chemohormonal therapy to stereotactic ablative radiotherapy of oligometastases. Urol Oncol 2016; 34:225-32.

11. Palma D, Olson R, Harrow S, et al. Stereotactic ablative radiotherapy versus standard of care palliative treatment in patients with oligometastatic cancers (SABR-COMET): A randomised, phase 2, open-label trial. Lancet 2019; 393:2051-8.

12. Ost P, Reynders D, Decaestecker K, A et al. Surveillance or metastasis-directed therapy for oligometastatic prostate cancer recurrence: A prospective, randomized, multicenter phase II trial. J Clin Oncol 2018; 36:446-53.

13. Phillips R, Lim SJ, Shi WY, Antonarakis ES, Rowe S, Gorin M, Deville C, Greco SC, Denmeade S, Paller C, DeWeese TL. Primary outcomes of a phase II randomized trial of Observation versus stereotactic ablative RadiatIon for OLigometastatic prostate CancEr (ORIOLE). International Journal of Radiation Oncology • Biology• Physics 2019; 105:681 


\section{Figures and Tables}

Fig. 1. Frequency of clinical measures of treatment response used for therapeutic guidance (ranked from $1^{\text {st }}$ to $5^{\text {th }}$ in order of importance; percentage of respondents). CT: computed tomography; MRI: magnetic resonance imaging; PSA: prostate-specific antigen.

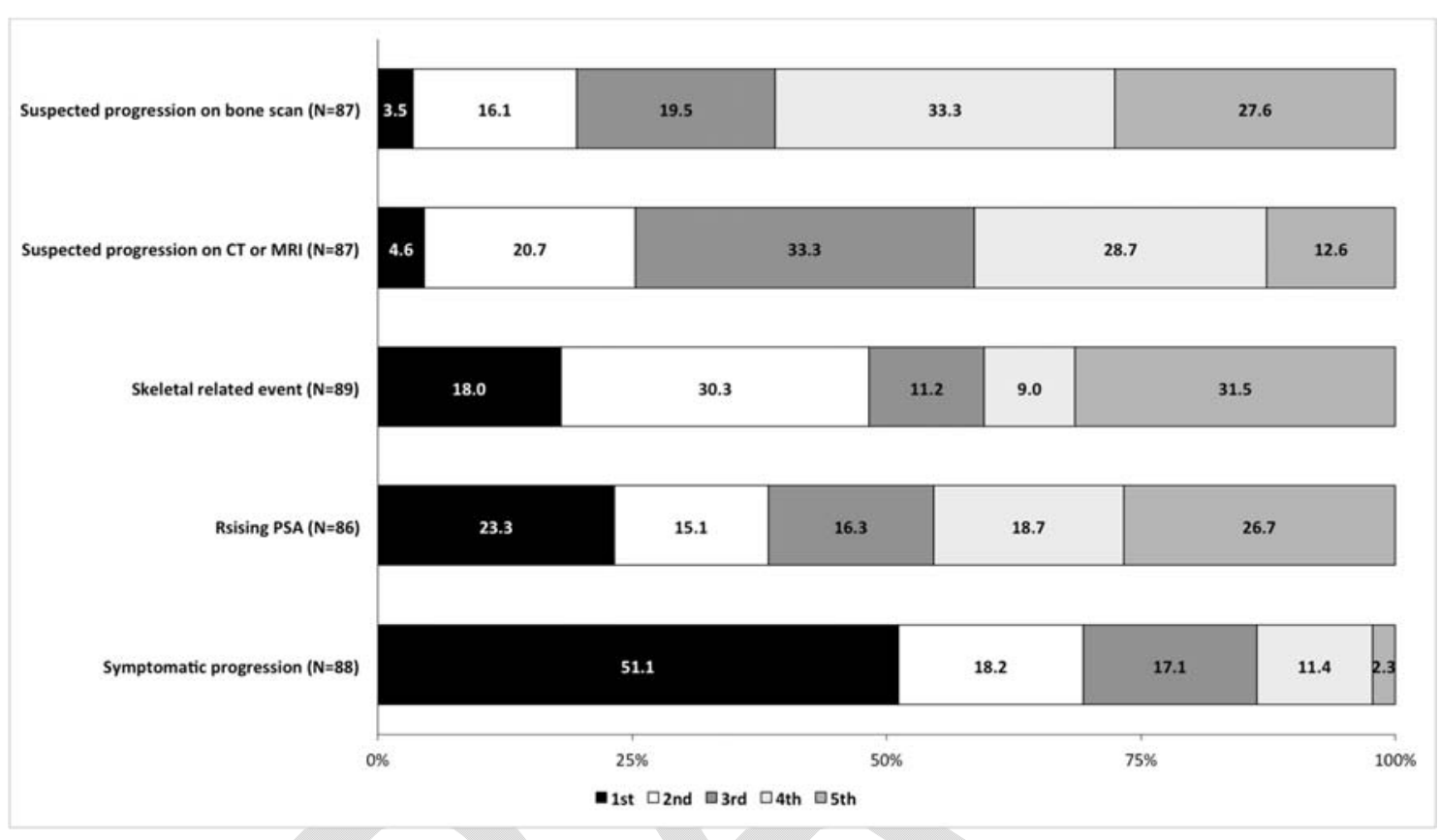

\begin{tabular}{|c|c|}
\hline \multicolumn{2}{|c|}{$\begin{array}{l}\text { Table } 1 \text {. Use of bone scans in the management of metastatic castrate resistant } \\
\text { prostate cancer }\end{array}$} \\
\hline $\begin{array}{l}\text { Is a baseline bone scan performed when initiating new } \\
\text { treatments? }(\mathrm{N}=87)\end{array}$ & n (\%) \\
\hline No & $5(5.7 \%)$ \\
\hline Yes & $82(94.3 \%)$ \\
\hline \multicolumn{2}{|l|}{$\begin{array}{l}\text { How often are scheduled bone scans performed on asymptomatic } \\
\text { patients receiving treatment }(\mathrm{N}=89)\end{array}$} \\
\hline No scheduled bone scan & $46(51.7 \%)$ \\
\hline Every $3-4$ months & $2(2.2 \%)$ \\
\hline Every 6 months & $12(13.5 \%)$ \\
\hline Every 12 months & $11(12.4 \%)$ \\
\hline Frequently depending on PSA kinetics & $18(20.2 \%)$ \\
\hline \multicolumn{2}{|l|}{$\begin{array}{l}\text { Does the type of therapy affect the frequency of obtaining a bone } \\
\text { scan? }(\mathrm{N}=82)\end{array}$} \\
\hline No & $77(93.9 \%)$ \\
\hline Increase frequency if treatment with radium 223 & $5(6.1 \%)$ \\
\hline $\begin{array}{l}\text { Is a bone scan progression confirmed with additional imaging? } \\
(\mathrm{N}=88)\end{array}$ & \\
\hline
\end{tabular}




\begin{tabular}{|l|l|}
\hline No & $46(52.3 \%)$ \\
\hline CT only & $26(29.5 \%)$ \\
\hline CT and/or MRI & $11(12.5 \%)$ \\
\hline CT and/or X-ray & $3(3.4 \%)$ \\
\hline MRI only & $2(2.3 \%)$ \\
\hline $\begin{array}{l}\text { Is a suspected progression in bone scan (within 12 weeks of } \\
\text { starting a new treatment) confirmed with a repeat bone scan? } \\
(\mathrm{N}=89)\end{array}$ & \\
\hline No & $60(67.4 \%)$ \\
\hline If yes, when? & $16(18.0 \%)$ \\
\hline $2-3$ months & $4(4.5 \%)$ \\
\hline 4-6 months & $9(10.1 \%)$ \\
\hline Depends on symptoms PSADT, clinical trial requirement & \\
\hline
\end{tabular}

CT: computed tomography; MRI: magnetic resonance imaging; PSA: prostate-specific antigen; PSADT: PSA doubling time. 